\title{
Nuclear phenomena derived from quark-gluon strings
}

Bohr, Henrik; Providencia, Constanca; Providencia, Joao da

Published in:

Physical Review C

Link to article, DOI:

10.1103/PhysRevC.71.055203

Publication date:

2005

Document Version

Publisher's PDF, also known as Version of record

Link back to DTU Orbit

Citation (APA):

Bohr, H., Providencia, C., \& Providencia, J. D. (2005). Nuclear phenomena derived from quark-gluon strings. Physical Review C, 71(5), 055203. https://doi.org/10.1103/PhysRevC.71.055203

\section{General rights}

Copyright and moral rights for the publications made accessible in the public portal are retained by the authors and/or other copyright owners and it is a condition of accessing publications that users recognise and abide by the legal requirements associated with these rights.

- Users may download and print one copy of any publication from the public portal for the purpose of private study or research.

- You may not further distribute the material or use it for any profit-making activity or commercial gain

- You may freely distribute the URL identifying the publication in the public portal

If you believe that this document breaches copyright please contact us providing details, and we will remove access to the work immediately and investigate your claim 


\title{
Nuclear phenomena derived from quark-gluon strings
}

\author{
Henrik Bohr, ${ }^{1}$ Constança Providência, ${ }^{2}$ and João da Providência ${ }^{2}$ \\ ${ }^{1}$ Department of Physics, B. 307, Danish Technical University, DK-2800 Lyngby, Denmark \\ ${ }^{2}$ Departamento de Física, Universidade de Coimbra, P-3000 Coimbra, Portugal
}

(Received 14 September 2004; revised manuscript received 15 March 2005; published 20 May 2005)

\begin{abstract}
We propose a quantum chromodynamics (QCD) based many-body model for the nucleus where the strong coupling regime is controlled by a three-body string force, and the weak coupling regime is dominated by a pairing force. This model operates effectively with a quark-gluon Lagrangian containing a pairing force from instantons, and a baryonic string term which contains a confining potential. The unified model for weak and strong coupling regimes is, however, only consistent at the border of perturbative QCD. The baryonic string force is necessary, as a stability and compressibility analysis shows, for the occurrence of the phases of nuclear matter. The model exhibits a quark deconfinement transition and chiral restoration, which are ingredients of QCD and give qualitatively correct numerics. The effective model is shown to be isomorphic to the Nambu-Jona-Lasinio model and exhibits the correct chirality provided that the chiral fields are identified with the two-particle strings, which are natural in a QCD framework. Moreover, the model is able to reconcile qualitatively such aspects of hadronic physics as saturation density and binding energy of nuclear matter, surface density of finite nuclei, mass of the scalar particle, medium range $N N$ interaction, and $f_{\pi}$ value in vacuum.
\end{abstract}

DOI: 10.1103/PhysRevC.71.055203

PACS number(s): 24.85.+p, 11.10.Ef, 21.65.+f, 21.30.Fe

\section{INTRODUCTION}

It has long been an important task for nuclear physicists to accommodate the most accepted fundamental quark interaction, quantum chromodynamics (QCD), in a field theoretical setting. This is because the nucleus constitutes the most important observational bound state system governed by the strong interaction. Furthermore, since the nucleons, the elements of the nuclear bound state, are believed to consist of quarks for which the rigorous strong interaction field theory applies, it is natural to try to describe the nucleus in terms of quarks (and gluons) along with nucleons. The present paper therefore aims at explaining nuclear properties and structure in terms of quarks and gluons. Still, one could be skeptical about using quarks with no direct observables in the description of nuclear physics that is very much based on experimental data. However, since the occurrence of strangeness excitations in hypernuclear physics needs to be explained by isospin or flavor quantum numbers that naturally come out of a quark model, there are good arguments for including quarks in nuclear physics.

There has been the obstacle of having a strong quark coupling constant above unity in the interesting range of the strong interaction which applies to the bound state and resonance phenomena. This strong coupling regime is characterized by low momentum transfer and corresponds to the region where quarks are believed to be confined within the hadrons, contrary to the high momentum transfer behavior in the region where the coupling is small and quarks are believed to be free. A coupling constant above unity in the region of the strong interaction that is interesting for nuclear physics will basically render a perturbative description of the strong interaction field theory useless. We therefore use the trick of putting in quark clustering by hand, so that nucleons are treated as elementary particles, but quark structure is taken into account in the interactions by assuming that the mass of the nucleon depends on the medium through the local values of the fields $\sigma, \vec{\pi}$. Once that is done, we try to perturb the energy around the solution so obtained. We also appeal to similar phenomena in other areas of physics (i.e., fluid dynamics) whose solutions can be applied to the nuclear system.

In the beginning of the paper, we introduce the quark description of the nuclear system and then propose a many-body Lagrangian for the nucleus. We continue with a description inspired by fluid dynamics and try to derive phases that could be relevant for nuclear physics. It is our aim to develop a model which is able to reconcile, at least qualitatively, such aspects of hadronic physics as saturation density and binding energy of nuclear matter, surface density of finite nuclei, mass of the scalar particle, medium range $N N$ interaction, and $f_{\pi}$ value in vacuum.

\section{A QUARK MODEL FOR NUCLEAR STRUCTURE}

In the following chapters, we propose a general Lagrangian for the nuclear system in terms of quarks and gluons that is based on an interaction like that of gauge theories in the strong interaction version of quantum chromodynamics, QCD.

We then describe the Lagrangian as a chiral model, but we first list the various ingredients as they appear in the energy functional of the model.

\section{A. The pairing force}

A starting point for a QCD model for the nucleus in terms of quarks and gluons could be a kind of a bag model in analogy to the well known Massachusetts Institute of Technology (MIT) model [1] for baryons and mesons, but bearing in mind that the nucleons themselves are MIT bags. In the usual setting of the MIT model, there is a strong influence of phenomenological 
aspects that we eventually try to derive with field theoretical interaction terms.

The generic form of the MIT bag model operates with a back pressure that ensures that the quarks are confined to the bag and there is a Coulomb force between the quarks. In the case where the entire nucleus is to be considered as an MIT bag with quarks moving within the boundaries of the bag, the bag pressure is again an infinite potential well that keeps the quarks confined to the nucleus. The quarks are, as usual, fermions with flavor and color charges. As for the interaction between the quarks, a crucial pairing force between the isospins ensures that the total isospin is zero. In the next subsection, we introduce a baryonic string force that is responsible for nucleon formation and also for the bag pressure.

The pairing force which has been derived from instanton contributions in QCD field theory [2] is able to account for important features of nuclear structure. For the one-instanton contribution, the pairing force is truly a two-body force. Its expression is

$$
P_{2}=g \bar{\psi}_{i}(x)\left(1+i \gamma_{5}\right) \psi_{i \prime}(x) \bar{\psi}_{j}(x)\left(1+i \gamma_{5}\right) \psi_{j^{\prime}}(x) \varepsilon_{i{ }^{\prime} j^{\prime}} \varepsilon_{i j},
$$

where $\varepsilon_{i j}$ antisymmetrizes the isospin indices $i, j$.

In the color zero sector, this effective interaction can be reduced to the Nambu-Jona-Lasinio (NJL) form [3,4], $\beta_{1} \beta_{2}\left[I_{1} I_{2}-\left(\vec{\tau}_{1} \gamma_{1}^{5}\right) \cdot\left(\vec{\tau}_{2} \gamma_{2}^{5}\right)\right]$. In that case, the missing terms, which constitute the difference between both expressions

$$
\begin{aligned}
\beta_{1} \beta_{2}\left\{\left(I_{1}+i \gamma_{1}^{5}\right)\left(I_{2}+i \gamma_{2}^{5}\right)\left(I_{1} I_{2}+\vec{\tau}_{1} \cdot \vec{\tau}_{2}\right)\right. \\
\left.-\left[I_{1} I_{2}-\left(\vec{\tau}_{1} \gamma_{1}^{5}\right) \cdot\left(\vec{\tau}_{2} \gamma_{2}^{5}\right)\right]\right\},
\end{aligned}
$$

average out to zero in a mean field approach.

\section{B. The baryonic string force in the nuclear bag}

In this subsection, we discuss the many-body string force term. We therefore introduce a three-body string force to the Hamiltonian. This force replaces the MIT bag pressure and is supposed to be responsible for nucleon bag formation. The string force is proportional to the distance between the quarks and is a very dominating force, so perturbation theory with the string force as a perturbation is not possible. On the other hand, the strings will triplet-wise neutralize the color, leaving us with only dipole or quadrupole moments. The string force is a qualified guess as to a configuration-an ansatz.

We now specify the baryonic string configuration. We have only limited information about nonperturbative string configurations. Lattice QCD gives, in the strong coupling limit, an indication of color tube strings between two heavy quarks. To make a consistent picture of the pairing and baryonic forces, we are staying within the border of perturbation theory, with the strong coupling constant $\alpha_{\mathrm{QCD}}$ being around 1 .

We can, however, resort to the dual string theories for help concerning string parameters. Here, the string interaction is proportional to the minimal length $L$ of the string, so that

$$
V_{\mathrm{st}}=\frac{1}{2 \pi \alpha^{\prime}} L=\sqrt{\sigma} L,
$$

where $\alpha^{\prime}$ is the Regge intercept, and $\sigma$ is the string tension. In the sequel, to avoid confusion with the $\sigma$ field, we write

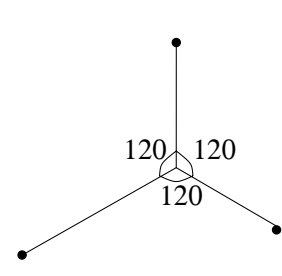

(a)

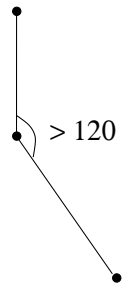

(b)

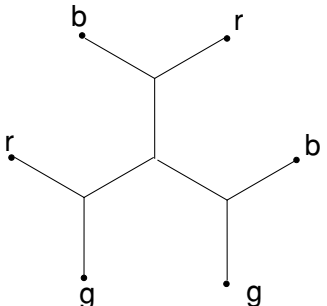

(c)
FIG. 1. Baryonic string configurations. (a) The angles of the triangle defined by a three-quark cluster are all less than $2 \pi / 3$. (b) One of the angles of the triangle defined by a three-quark cluster is greater than $2 \pi / 3$. (c) A six-quark cluster. This configuration is here included as a possibility but not used in the full picture. It will be discussed in a forthcoming paper.

$\kappa=\sqrt{\sigma}$. The total three-body force is then, apart from color 0 projection operators,

$$
V_{3}=\int d x_{1} d x_{2} d x_{3} \bar{\psi}\left(x_{1}\right) \bar{\psi}\left(x_{2}\right) \bar{\psi}\left(x_{3}\right) V_{\mathrm{st}} \psi\left(x_{3}\right) \psi\left(x_{2}\right) \psi\left(x_{1}\right) .
$$

The two possible [5] configurations between three quarks in $S U(3)-\mathrm{QCD}$ when we require $S U(3)$ gauge invariance are shown in Fig. 1. This means that quarks cluster in multiples of three. Within our approximation, we consider only two- and three-body forces arising in the color neutral configurations [see Fig. 1 (a, b)].

Mesons are, as usual, strings between a quark-antiquark pair.

\section{The chiral aspects of the Lagrangian}

In this subsection, we briefly mention relevant aspects of chiral symmetry, which we insist on having properly implemented. This is important because one of our aims is to describe chiral symmetry restoration in hadronic matter. The pairing force is chirally symmetric. On the other hand, following the procedure of Ref. [8], the string interaction, which is not automatically chirally invariant, may be also reconciled with chiral symmetry. In our approach, the Dirac see is naturally responsible for the dynamical breaking of chiral symmetry, contrary to an artificial device such as a Mexican hat. Our calculation shall be based on effective Lagrangians, of either the Nambu-Jona-Lasinio (NJL) or the $\sigma$ model type. We argue that the two models are equivalent as far as the mean field description of bulk static properties of hadronic matter is concerned. However, they lead, in the random phase approximation (RPA) approximation, to manifestly distinct dynamics, in spite of being equally consistent with chiral symmetry, so that it really matters which model one considers if, beyond bulk properties, we are interested in the surface properties or the response of the system to an external probe.

\section{THE EFFECTIVE ACTION AND THE DIRAC EQUATIONS}

In this section, we describe the general structure of the effective action which is relevant for the present development, 
and we try to motivate, on the basis of the Dirac equation of quark dynamics, the model that is considered in the next section. The effective interaction is supposedly obtained by integrating over the gauge fields.

Below, we describe all the terms appearing in the effective Lagrangian, where the potential terms come out of a typical cluster expansion in the quark fields. First comes the kinetic term $i \bar{\psi} \gamma^{\mu} \partial_{\mu} \psi$. Next come all the four-fermion terms making up the two-body force,

$$
\begin{gathered}
\bar{\psi} \psi \bar{\psi} \psi+(\bar{\psi} \vec{\tau} \psi)(\bar{\psi} \vec{\tau} \psi)-\left(\bar{\psi} \gamma_{5} \psi\right)\left(\bar{\psi} \gamma_{5} \psi\right) \\
-\left(\bar{\psi} \vec{\tau} \gamma_{5} \psi\right) \cdot\left(\bar{\psi} \vec{\tau} \gamma_{5} \psi\right),
\end{gathered}
$$

which, following a common procedure, we, for simplicity, replace by the combination which is familiar from the NJL model [3],

$$
\bar{\psi} \psi \bar{\psi} \psi-\left(\bar{\psi} \vec{\tau} \gamma_{5} \psi\right)\left(\bar{\psi} \vec{\tau} \gamma_{5} \psi\right)
$$

Finally come the six-quark terms contributing to the threebody string force. In order to insure chiral invariance, these three-body forces can be understood as reductions of appropriate chiral invariant four-body forces.

It is assumed that the string force is responsible for the transformation of the quark degrees of freedom into nucleon and $\sigma, \vec{\pi}$ meson degrees of freedom, in the strong coupling limit, at low energies. The $\sigma$-nucleon coupling may be taken to be three times as large as the $\sigma$-quark coupling. However, as shown in Refs. [6,7], the quark dynamics arising from the confining force has the consequence that the nucleon coupling constant decreases as the baryonic density increases. It is argued below that this effect is important for saturation of nuclear matter.

In Ref. [6], the confinement of quarks into nucleons is described on the basis of the MIT bag model through the solution of an appropriate Dirac equation,

$$
i \partial_{t} \psi=-i \boldsymbol{\alpha} \cdot \boldsymbol{\nabla} \psi+V_{\mathrm{MIT}} \psi+g \beta \sigma \psi .
$$

The $\sigma$ field is determined self-consistently, its source being the scalar density due to $\psi$. If the MIT interaction $V_{\text {MIT }}[1]$ is replaced by a string force $m(r)=\kappa r$, where $\kappa^{2}$ is the string tension, in a form which is consistent with chiral symmetry, the previous equation may be replaced by [8]

$$
i \partial_{t} \psi=-i \boldsymbol{\alpha} \cdot \nabla \psi+\left[f_{\pi}^{-1} m(r)+g\right]\left(\sigma-i \gamma_{5} \vec{\tau} \vec{\pi}\right) \psi,
$$

where $\sigma, \vec{\pi}$ denote, respectively, the $\sigma$ meson and the pion field, and $f_{\pi}$ is the pion decay constant in the vacuum. Here, we have already factored out the three-body wave function into a product described by three independent equations. There are important differences between the models in Refs. [6] and [8] which explain the extra factors we introduce, namely $\left[f_{\pi}^{-1} m(r)+g\right]$, and which are of interest for the present discussion, especially due to the fact that we insist on having chiral invariance. Moreover, the $\sigma$ field in our case couples to positive and negative energy states, and therefore is nonzero in the vacuum. The relevant consequence of this, which is explained below with respect to the phase analysis, is that we expect a much stronger saturation effect at a high density of hadronic matter, and hence an enhancement of asymptotic freedom phenomena for quarks.
We now check the consistency of our string configuration and the string potential $m(r)$ by analyzing the spectrum of the Dirac operator,

$$
\left\{\alpha_{r} p_{r}+\frac{i}{r} \alpha_{r} \beta k+\beta \sigma\left[f_{\pi}^{-1} m(r)+g\right]\right\} \psi=\varepsilon(\sigma) \psi,
$$

where

$$
\alpha_{r}=\left[\begin{array}{cc}
0 & -i \\
i & 0
\end{array}\right], \quad \beta=\left[\begin{array}{cc}
1 & 0 \\
0 & -1
\end{array}\right],
$$

and $k= \pm 1, \pm 2, \cdots$. Here, we have used spherical symmetry. We take, for $r<1 \mathrm{fm}, g+f_{\pi}^{-1} m(r)=\kappa f_{\pi}^{-1} r$, where $\kappa$ is the same as defined in connection with Eq. (1). This gives us

$$
\begin{gathered}
\psi(r)=\frac{1}{r}\left[\begin{array}{c}
F(r) \\
G(r)
\end{array}\right], \\
{\left[\varepsilon(\sigma)-\sigma f_{\pi}^{-1} \kappa r\right] F+\frac{d G}{d r}+\frac{k}{r} G=0,} \\
{\left[\varepsilon(\sigma)+\sigma f_{\pi}^{-1} \kappa r\right] G-\frac{d F}{d r}+\frac{k}{r} F=0 .}
\end{gathered}
$$

Eliminating one of the variables, we obtain a second order equation in the other which may be solved numerically. The equations will have the form

$$
\begin{aligned}
& \left(1+\frac{\sigma f_{\pi}^{-1} \kappa}{\varepsilon^{2}} \rho\right) G+\left(\frac{d}{d \rho}-\frac{k}{\rho}\right) \\
& \times\left(1-\frac{\sigma f_{\pi}^{-1} \kappa}{\varepsilon^{2}} \rho\right)^{-1}\left(\frac{d}{d \rho}+\frac{k}{\rho}\right) G=0,
\end{aligned}
$$

and similarly for $F$. We have defined $\rho=\varepsilon r$.

A few qualitative observations can now be made on the basis of Eq. (10). This equation is singular for $\sigma f_{\pi}^{-1} \kappa= \pm \varepsilon^{2}$. This gives certain rules for the energy eigenvalue $\varepsilon(\sigma)$. It should be noticed that the string interaction is proportional to the $\sigma$ field.

The QCD vacuum, which is studied in these calculations, is a very complex object, not least because of the strong contributions from both positive and negative energy states. In our model, it is described as a collection of nucleonic bags arising out of the solution of the Dirac equation, in all possible momentum states $P$ such that $|P| \leqslant \Lambda$, where $\Lambda$ is a regularizing cutoff. In the framework of the independent particle approach, which has been described, the mass of the nucleonic bag is approximately $M(\sigma)=3 \varepsilon(\sigma)$. The scalar density $\rho_{S}$ and the energy of the vacuum are also important quantities, and their calculation, which is given in Sec. IV, is very informative concerning this point. The vacuum value of the $\sigma$ field is set to $f_{\pi}$, i.e., $\sigma=f_{\pi}$ if the Fermi momentum vanishes $\left(p_{F}=0\right)$.

The asymptotic solution for $G$ and $F$ fields in the Dirac equation can easily be found by approximating the equation to

$$
G-\frac{\varepsilon^{2}}{\kappa \rho} \frac{d}{d \rho}\left(\frac{\varepsilon^{2}}{\kappa \rho} \frac{d}{d \rho} G\right)=0 .
$$

Thus, the asymptotic solution for $G$ and $F$ will be

$$
G \sim \exp \left(-\frac{\kappa \rho^{2}}{2 \varepsilon^{2}}\right)
$$


Here, we can easily read out the size of the nucleon bag to be $1 / \sqrt{\kappa}$, which becomes approximately $0.8 \mathrm{fm}$, in vacuum.

\section{THE MODEL}

To be more specific, we present now in greater detail the basic scheme which supports our calculation. This framework is motivated by the previous discussion and is based on the assumption that quarks and gluons are the building blocks of nucleons. We assume that the interaction between quarks originates from an instanton force and from a confining string interaction, the latter being responsible for the clustering of quarks into nucleons, and the former for the emergence of a $\sigma$ field which mediates the interaction between nucleons and is understood as a quark-antiquark disturbance of the vacuum. According to this picture, the well known $\sigma$ model Lagrangian density may be extended to read

$$
\begin{aligned}
\mathcal{L}= & i \bar{\psi} \gamma^{\mu} \partial_{\mu} \psi-g_{s}(\sigma, \vec{\pi})\left[(\bar{\psi} \psi) \sigma+i\left(\bar{\psi} \gamma^{5} \vec{\tau} \psi\right) \cdot \vec{\pi}\right] \\
& +g_{v}\left(\bar{\psi} \gamma_{\mu} \psi\right) \omega^{\mu}-\frac{1}{2} m_{\sigma}^{2}\left(\sigma^{2}+\vec{\pi}^{2}\right)+\frac{1}{2} m_{v}^{2} \omega_{\mu} \omega^{\mu} \\
& +\frac{1}{2}\left(\partial^{\mu} \sigma \partial_{\mu} \sigma+\partial^{\mu} \vec{\pi} \partial_{\mu} \vec{\pi}\right)-\frac{1}{4} \omega^{\mu \nu} \omega_{\mu \nu},
\end{aligned}
$$

where $\psi$ denotes the nucleon field (not the quark field). This Lagrangian density describes an assembly of nucleons, regarded as composite particles, which interact with $\sigma, \vec{\pi}$ and $\omega^{\mu}$ fields. The familiar Mexican hat does not appear in Eq. (11). However, terms involving the $\omega$ field are introduced, as in the Walecka model [9]. In a conventional model, with elementary particles, $g_{s}$ is a constant. In our model, which we call the extended $\sigma$ model (ESM), the composite nature of the particles reflects itself in the fact that $g_{s}$ depends on the local values of the fields $\vec{\pi}, \sigma$. We assume that

$$
\begin{aligned}
g_{s}(\sigma, \vec{\pi}) & =g_{0} \sqrt{\frac{1-\sqrt{1-4 g_{0}^{2} a^{2}\left(\sigma^{2}+\vec{\pi}^{2}\right)}}{2 g_{0}^{2} a^{2}\left(\sigma^{2}+\vec{\pi}^{2}\right)}} \\
& \approx g_{0}\left[1+\frac{1}{2} g_{0}^{2} a^{2}\left(\sigma^{2}+\vec{\pi}^{2}\right)\right]
\end{aligned}
$$

where $a, g_{0}$ are phenomenological parameters. For consistency, we require $\left|2 g_{0} a \sigma\right| \ll 1$. For an extended system, $\vec{\pi}=0$. The dynamically generated mass of the nucleon becomes then $M(\sigma)=g_{s}(\sigma) \sigma$. From Eq. (12), it follows that

$$
M(\sigma)=g_{0} \sigma \sqrt{\frac{1-\sqrt{1-4 g_{0}^{2} a^{2} \sigma^{2}}}{2 g_{0}^{2} a^{2} \sigma^{2}}} \approx g_{0} \sigma\left(1+\frac{1}{2} g_{0}^{2} a^{2} \sigma^{2}\right) .
$$

We have circumvented the laborious problem of determining the mass $M(\sigma)$ of a nucleon subject to an external field $\sigma$ by making an ansatz. According to our assumption, $M(\sigma)$ behaves as $g_{0} \sigma$ for small $\sigma$, but increases faster for large $\sigma$. We also have

$$
\sigma^{2}=\frac{M^{2}}{g_{0}^{2}}\left(1-a^{2} M^{2}\right)
$$

The energy of an assembly of nucleons is

$$
\begin{aligned}
\mathcal{E}(\sigma, \omega)= & -\eta \sum_{p_{F} \leq|\boldsymbol{P}| \leq \Lambda} \sqrt{\boldsymbol{P}^{2}+g_{s}^{2} \sigma^{2}}-\eta \sum_{|P| \leq p_{F}} g_{v} \omega \\
& +\frac{1}{2} m_{\sigma}^{2} \sigma^{2} V-\frac{1}{2} m_{v}^{2} \omega^{2} V,
\end{aligned}
$$

or, after eliminating $\sigma$ in favor of $M$ and "minimization" with respect to $\omega$,

$$
\begin{aligned}
\mathcal{E}(\sigma)= & -\eta \sum_{p_{F} \leq|P| \leq \Lambda} \sqrt{P^{2}+M^{2}}+\frac{m_{\sigma}^{2}}{2 g_{0}^{2}} M^{2} \\
& \times\left(1-a^{2} M^{2}\right) V+\frac{g_{v}^{2} p_{F}^{6} \eta^{2}}{2^{3} 3^{2} m_{v}^{2} \pi^{4}} V .
\end{aligned}
$$

Here, $V$ is the normalization volume, $p_{F}$ is the Fermi momentum, $\Lambda$ is the regularizing cutoff momentum, and $\eta=2 N_{f}$ is the degeneracy. The natural assumption has been made that the wave function of the assembly of nucleons is a Slater determinant. Minimizing $\mathcal{E}(\sigma)$, we obtain the generalized gap equation

$$
\frac{g_{0}^{2} \eta}{m_{\sigma}^{2} V} \sum_{p_{F} \leq|P| \leq \Lambda} \frac{1}{\sqrt{P^{2}+M^{2}}}=1-2 a^{2} M^{2} .
$$

The coupling constant depends on $\sigma$, and, through $\sigma$, on the baryonic density. The coupling constant increases essentially linearly with $\sigma^{2}$. The practical consequence of such a behavior is the emergence of a rapid increase of the energy with density, tantamount to the onset of a repulsive contribution. This behavior plays a decisive role in fixing the saturation density.

\section{NJL VERSUS $\sigma$ MODEL}

As mentioned in Sec. II, the quark nuclear model we propose incorporates, along with the string force, the usual NJL model with the characteristic four-fermionic term, actually coming out of an instanton gas. In this section, we discuss briefly the equivalence of the $\sigma$ model to the NJL model [3], as far as the mean field description of bulk properties of hadronic matter is concerned. The NJL model is defined by the Lagrangian density

$$
\mathcal{L}=\bar{\psi}\left(i \gamma^{\mu} \partial_{\mu}\right) \psi+\frac{G_{S}}{2}\left[(\bar{\psi} \psi)^{2}+\left(\bar{\psi} i \gamma_{5} \vec{\tau} \psi\right)^{2}\right] .
$$

The $\sigma$ model is defined by Eq. (11), provided we replace the coupling parameter $g_{s}(\sigma, \vec{\pi})$ by a constant. A regularizing momentum cutoff $\Lambda$ is part of both models. Using standard procedures, the Lagrangian (16) leads to the Hamiltonian

$$
\begin{aligned}
\mathcal{H}_{\mathrm{NJL}}= & \sum_{k=1}^{N} \boldsymbol{P}_{k} \cdot \boldsymbol{\alpha}_{k}+\frac{G_{S}}{2} \sum_{k, l=1}^{N} \delta\left(\boldsymbol{r}_{k}-\boldsymbol{r}_{l}\right) \\
& \times \beta_{k} \beta_{l}\left(1-\gamma_{k}^{5} \gamma_{l}^{5} \vec{\tau}_{k} \vec{\tau}_{l}\right) .
\end{aligned}
$$

The vacuum is described by a Slater determinant $\left|\Phi_{0}\right\rangle$ created by the operators $b_{\boldsymbol{P},-}^{\dagger}$, satisfying $|\boldsymbol{P}|<\Lambda$, associated with plane wave negative energy eigenstates of the single particle Hamiltonian $h=\boldsymbol{P} \cdot \boldsymbol{\alpha}+\beta M$, and by the operators $b_{\boldsymbol{P},+}^{\dagger}$, 
satisfying $|\boldsymbol{P}|<p_{F}$, so that $p_{F}$ is the Fermi momentum, associated with positive energy eigenfunctions of the same operator. The nucleon "constituent mass" $M$ is a variational parameter.

The energy expectation value $\mathcal{E}=\left\langle\Phi_{0}\left|\mathcal{H}_{\mathrm{NJL}}\right| \Phi_{0}\right\rangle$ reads

$$
\begin{aligned}
\mathcal{E} & =-\eta \sum_{|\boldsymbol{P}|=p_{F}}^{\Lambda} \frac{P^{2}}{\varepsilon_{P}}-\frac{G_{S}}{2 V}\left[\eta \sum_{|\boldsymbol{P}|=p_{F}}^{\Lambda} \frac{M}{\varepsilon_{P}}\right]^{2} \\
& =-\frac{\eta V}{2 \pi^{2}} \int_{p_{F}}^{\Lambda} d P \frac{P^{4}}{\varepsilon_{P}}-\frac{G_{S} V}{2}\left[\frac{\eta}{2 \pi^{2}} \int_{p_{F}}^{\Lambda} d P \frac{M P^{2}}{\varepsilon_{P}}\right]^{2},
\end{aligned}
$$

where $\varepsilon_{P}=\sqrt{P^{2}+M^{2}}, V$ is the normalization volume, $\eta$ is the degeneracy, and for nuclear matter, $G_{S}$ is 9 times bigger than the corresponding quark matter value. For quark matter, $\eta=2 N_{c} N_{f}$, while for nuclear matter, $\eta=2 N_{f}$. The condition $\partial \mathcal{E} / \partial M=0$ leads to the gap equation

$$
1=\frac{\eta G_{S}}{V} \sum_{|P|=p_{F}}^{\Lambda} \frac{1}{\varepsilon_{P}}=\frac{\eta G_{S}}{2 \pi^{2}} \int_{p_{F}}^{\Lambda} d P \frac{P^{2}}{\varepsilon_{P}}
$$

which fixes $M$. For nuclear matter, we fix $\Lambda$ so that $M=$ $939 \mathrm{MeV}$. For quark matter, we fix $\Lambda$ so that $M=313 \mathrm{MeV}$. If we take

$$
G_{S}=\frac{9 g_{0}^{2}}{m_{\sigma}^{2}}
$$

this is essentially the same gap equation as (15), except that the quantity $1-2 a^{2} M^{2}$ in the right-hand side (rhs) of (15) is here replaced by 1 .

Notice also that the minimum of $\mathcal{E}$ given by Eq. (18) is the same as the minimum of $\mathcal{E}(\sigma)$ given by Eq. (14), if $a$ is set equal to 0 in the expression for $M(\sigma)$, Eq. (13).

An extended NJL (ENJL) model essentially equivalent to (11) is easily obtained and reads

$$
\begin{aligned}
\mathcal{L}_{\mathrm{ENJL}}= & \bar{\psi}\left(i \gamma^{\mu} \partial_{\mu}\right) \psi+\frac{G_{S}}{2}\left[(\bar{\psi} \psi)^{2}+\left(\bar{\psi} i \gamma_{5} \vec{\tau} \psi\right)^{2}\right] \\
& +\frac{K}{12}\left[(\bar{\psi} \psi)^{2}+\left(\bar{\psi} i \gamma_{5} \vec{\tau} \psi\right)^{2}\right]^{2} \\
& +\frac{G_{V}}{2}\left[\left(\bar{\psi} \gamma_{\mu} \psi\right)\left(\bar{\psi} \gamma^{\mu} \psi\right)\right] .
\end{aligned}
$$

A similar model has been studied in Ref. [13].
TABLE I. Parameters of ESMI and ESMII.

\begin{tabular}{lcccc}
\hline \hline & $g_{s}$ & $g_{v}$ & $\Lambda(\mathrm{MeV})$ & $a$ \\
\hline ESMI & 5.81 & 10.75 & 477 & 0.30 \\
ESMII & 8.12 & 13.16 & 387 & 0.25 \\
\hline \hline
\end{tabular}

\section{THE PHASES OF NUCLEAR MATTER AND QUARK CLUSTERING}

In order to get a good understanding of the various phases nuclear matter can exhibit at different energies, it is useful to introduce some descriptors for the various nuclear processes. We usually describe quantum field interactions in terms of the momentum transfer of the strong interaction scattering involving quarks and gluons. One can as well picture the energy per nucleon as a function of pressure.

There are two distinct transitions that are important in the nuclear phase diagram [10] when varying pressure or momentum transfer. One is the chiral restoration transition, mostly determined by the instanton pairing force $[2,11]$. Secondly, there is the quark deconfinement transition when the nuclear "fluid" changes from "water" to "metallic fluid." This is a consequence of the diminishing baryonic string force, predominantly due to the behavior of the effective coupling constant.

We wish now to calculate the energy per particle of nuclear matter as a function of the density. As pointed out above, our two main ingredients are the pairing force and the baryonic string force. We take these contributions separately since they apply mostly to different scales. The effect of the string force is to confine quarks within nucleons, while the pairing force comes from instantons.

We have considered two extreme parametrizations of the ESM, denoted ESMI and ESMII. The corresponding results are shown in Tables I and II and Figs. 2 and 3. The parameters of the model, $g_{s}, g_{v}$, and $a$, were chosen in order to reproduce the saturation properties of nuclear matter, namely, binding energy, saturation density, and effective mass at saturation. The compressibility and $f_{\pi}$ are outputs of the calculation. We are now able to make the plots of the energy per nucleon as a function of density, shown in Fig. 2. The dotted line applies to a quark gas described by the Lagrangian (16) with quarks. The coupling constant $G_{S}=2.14 / \Lambda^{2}$ was chosen so that $f_{\pi}=93 \mathrm{MeV}$ and $\Lambda=654 \mathrm{MeV}$. This cutoff reproduces the correct quark mass in vacuum $m_{q}=313 \mathrm{MeV}$. We see that the quark equation of state (EOS) exhibits no binding, although it shows a local minimum corresponding to chiral symmetry restoration. This occurs at around $0.2 \mathrm{fm}^{-3}$, which corresponds

TABLE II. Properties of nuclear matter according to ESMI and ESMII: binding energy, equilibrium density, incompressibility, pion decay constants, $f_{\pi}$ in vacuum, $f_{\pi}^{*}$ at saturation, chiral transition density, and deconfinement density.

\begin{tabular}{lcccccccc}
\hline \hline & $\begin{array}{c}E_{B} \\
(\mathrm{MeV})\end{array}$ & $\begin{array}{c}\rho_{0} \\
\left(\mathrm{fm}^{-3}\right)\end{array}$ & $M / M_{0}$ & $\begin{array}{c}K \\
(\mathrm{MeV})\end{array}$ & $\begin{array}{c}f_{\pi} \\
(\mathrm{MeV})\end{array}$ & $\begin{array}{c}f_{\pi}^{*} \\
(\mathrm{MeV})\end{array}$ & $\begin{array}{c}\rho_{\chi} \\
\left(\mathrm{fm}^{-3}\right)\end{array}$ & $\begin{array}{c}\rho_{\mathrm{de}} \\
\left(\mathrm{fm}^{-3}\right)\end{array}$ \\
\hline ESMI & -15.75 & 0.15 & 0.7 & 225 & 150 & 134 & 0.46 & 0.687 \\
ESMII & -15.75 & 0.148 & 0.587 & 240 & 108 & 66 & 0.3 & 0.36 \\
\hline \hline
\end{tabular}




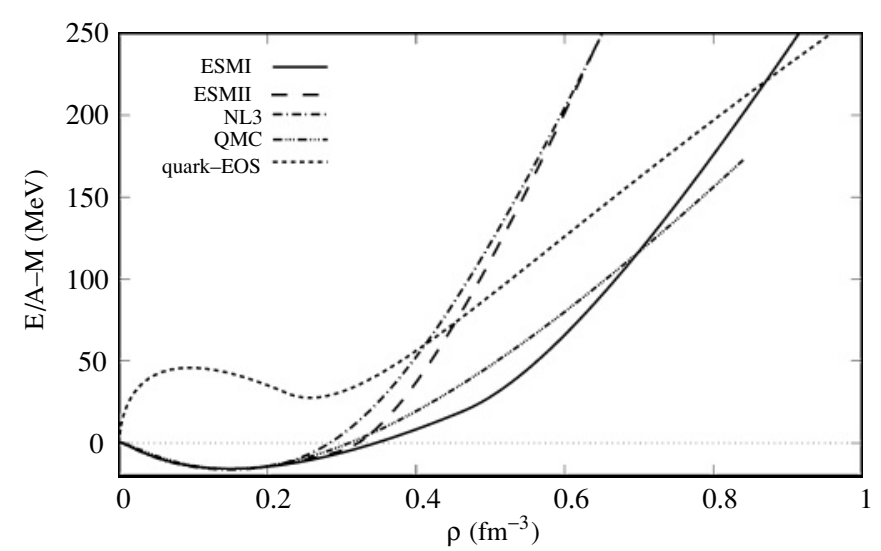

FIG. 2. Phase diagram of hadronic matter energy as a function of the density. The dotted line applies to a quark gas. The dashdotted curve corresponds to the so-called NL3 parametrization of the Walecka model [15]. The solid curve and the long-dashed curve present, respectively, the results of ESMI and ESMII. The dashed curve corresponds to the quark-meson coupling model [6].

to the generally accepted deconfinement transition expected at a temperature of about $200 \mathrm{MeV}$ in the vacuum. The solid curve and the long-dashed curve represent the effect of quark clustering according to ESMI and ESMII, respectively. The cutoff $\Lambda$ is such that chiral symmetry breaking of the vacuum reproduces the correct nucleon mass. The clear binding shown by these curves is due to the reduction in the kinetic energy arising from the clustering of three quarks into nucleons [12]. This latter behavior is crucial for stability. Our model takes into account not only the effect of quark clustering, but also the repulsion between the nucleonic bags. The interplay between these effects is described by Eqs. (11), (12), (14), (15). In Fig. 2, chiral symmetry restoration is shown as a discontinuity of the second derivative of the ESMI and ESMII curves. Deconfinement occurs close to the point where these curves intercept the quark-EOS dotted curve, being determined by the intercept of the pressure versus chemical potential curves. Although our calculation is indeed based on the ESM

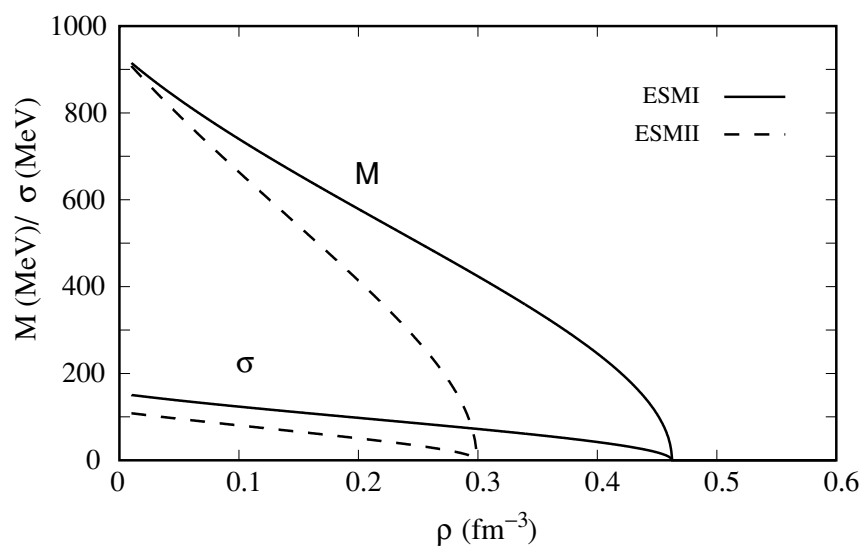

FIG. 3. Effective mass of the nucleon and $\sigma$ field as function of the density for ESMI and ESMII. Chiral symmetry restoration occurs when these quantities vanish.

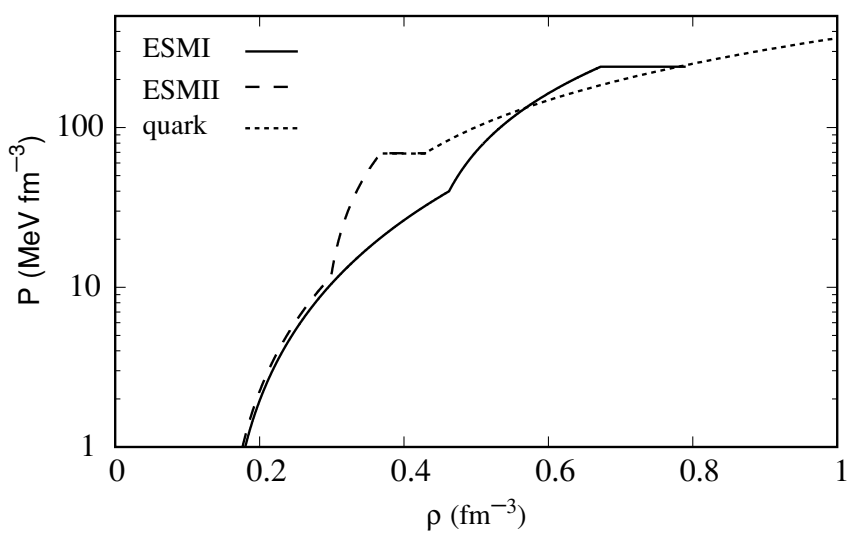

FIG. 4. Pressure versus density for ESMI and ESMII. Phase transitions are shown as discontinuities in the slopes of the curves.

[Eq. (11)], we observe that it would be equally possible to use the ENJL model [Eq. (20)], which also reflects the influence of the medium on the nucleon properties (see, for instance, Refs. [13] or [14]; in [14], a density dependent coupling is used, in contrast to our assumption of a coupling depending on the local value of the $\sigma$ field). However, we insist that the equivalence between the ESM [Eqs. (11), (12)] and ENJL models [Eq. (20)] applies only to the description of bulk properties. These models differ in the description of surface properties and in the predicted dynamics, such as the masses of scalar excitations, for which they lead to distinct dispersion relations. Also, for the value of $f_{\pi}$ in vacuum, both models lead to different expressions and quite distinct values.

It has been argued in Ref. [6] that the quark structure of the nucleon induces a mechanism for saturation by weakening, at high density, the attraction due to the $\sigma$ meson. We find that the contribution of this effect to the energy per particle is equivalent to a term essentially proportional to $\rho^{2}$. In order to fit the high energy scattering nuclear experiment, it is important that the slope in the high energy end (large pressure) not be too steep, meaning a fluid of nuclear matter that is not too incompressible.

In principle, one may expect to find two critical limits: the limit of chiral symmetry restoration, when the mass of the nucleon vanishes, and the deconfinement limit, when the baryonic string force becomes small and quarks get liberated. For ESMI, chiral symmetry restoration is depicted in Figs. 2, 3, and 4 at $\rho / \rho_{0} \approx 3.1$, while the transition from the confined phase to a deconfined phase is shown in Figs. 2 and 4 at $\rho / \rho_{0} \approx 4.5$. For ESMII, chiral symmetry restoration is depicted at $\rho / \rho_{0} \approx 2.0$, and the transition from the confined phase to a deconfined phase takes place at $\rho / \rho_{0} \approx 2.4$.

One may question the fact that the values of the cutoff or scale parameter $\Lambda$ for hadronic matter, $477 \mathrm{MeV}$ for ESMI and $387 \mathrm{MeV}$ for ESMII, are rather small. Indeed, they are only about $10 \%$ higher than the Fermi momentum for which the deconfinement transition is predicted to occur, namely $427 \mathrm{MeV}$ for ESMI and $324 \mathrm{MeV}$ for ESMII. However, these values for the Fermi momenta are obtained at the critical point for deconfinement. The scale parameter of the deconfined phase $\Lambda=654 \mathrm{MeV}$, is sufficiently large, even 
for considerably higher quark densities. On the other hand, using two different models for the confined phase and the deconfined phases is admittedly a rough approximation. Since a more rigorous treatment within a single model should be performed, our conclusions concerning deconfinement are of a qualitative nature. The present description of deconfinement is intended to give an order of magnitude of densities at which the transition will occur. Chiral symmetry restoration, which in our model occurs at a lower density, is not affected by these drawbacks.

We note that the equation of state predicted by ESMI is consistent with the one predicted by the quark-meson coupling model [6], and it is much softer than the one predicted by the so-called NL3 parametrization of the Walecka model [15]. The equation of state predicted by ESMII is also rather soft. Our model is consistent with the occurrence of a superfluid exotic phase of hadronic matter between the critical points corresponding to chiral symmetry restoration and deconfinement. In particular, this phase might be composed by Bose particles, each particle being constituted by six quarks. Our model is also consistent with the occurrence of the so-called color-flavor-locked phase, above the second critical point.

The values obtained for the pion decay constant, identified with the value of $\sigma$, constitute an interesting by-product of our model, being qualitatively reasonable. Indeed, for the parametrization ESMI, we find $f_{\pi}=150.5 \mathrm{MeV}$ in vacuum, and at saturation, we have $f_{\pi}=110.5 \mathrm{MeV}$. The value of the pion decay constant is sensitive to the values of the effective mass and in lesser degree, of the saturation density considered for fitting the model parameters. So, for the parametrization ESMII, we find $f_{\pi}=108 \mathrm{MeV}$ in vacuum, and at saturation, we have $f_{\pi}=66.3 \mathrm{MeV}$.

In the present treatment, we have only considered quark matter in the normal phase, since our main concern was the description of hadronic matter. It is well known, however, that the color superconducting [16] phase plays an important role in quark matter, considerably reducing its energy per quark. An excellent discussion of color superconductivity within the NJL model is presented in Ref. [17], where, for the two flavor case, a decrease of about $60 \mathrm{MeV}$ on the energy per particle is already predicted, according to figure 4.4 of that work. This effect is important because it will significantly reduce the Fermi momentum, pushing it away from the undesirable zone.

A comment on the considerable difference in behavior predicted by parametrizations ESMI and ESMII is in order. Parametrization EMSII is only of interest because it illustrates an extreme situation, almost at the border of applicability of our model, showing a more reasonable value of $f_{\pi}$ in vacuum, but at the cost of lower values of the cutoff $\Lambda$ and of the saturation (not transition) density $\rho_{0}$. In fact, the large difference in the behavior of the EOS of ESMI and II is not due to the $\Lambda$ value but to the large difference in the effective nucleon mass at saturation. A smaller effective nucleon mass at saturation means a chiral symmetry restoration at a smaller density and a stronger repulsive effect coming from the vector meson contribution at smaller densities. This same effect is also present in other relativistic hadronic models such as the nonlinear Walecka model.
Finally, we wish to mention a recent attempt pursued by Chanfray and collaborators [18] to reconcile the Walecka model with chiral symmetry, although along different lines.

\section{SUMMARY AND CONCLUSIONS}

In the present article, we have constructed a realistic quark model of nuclear structure phenomena which is inspired by the QCD quantum field theory, being formulated with very few free parameters, namely $\Lambda, a, g_{0}, g_{v}$. In its final form, our model resembles the conventional NJL model for nucleons with, however, consequential differences reflecting the influence of the medium on the properties of the nucleons. In order to meet important requirements of chiral symmetry arising in the description of nuclear phenomena at a subnuclear level, we have added to the starting Lagrangian, which describes the dynamics at the quark scale, important terms coming from chiral fields that appear at a higher order of QCD, in a cluster expansion sense. Such a unified model of nuclear physics is consistent with important data about nuclear phases from low energy physics and astrophysics [19], such as chiral symmetry restoration and quark deconfinement. The present model is able to reconcile qualitatively such aspects of hadronic physics as saturation density and binding energy of nuclear matter, surface density of finite nuclei, mass of the scalar particle, medium range $N N$ ineraction, and $f_{\pi}$ value in vacuum.

\section{ACKNOWLEDGMENTS}

We wish to thank Prof. K. Yazaki and Prof. J. Clark for helpful comments. J. P. wishes to thank S. Moszkowski, H. Walliser, Y. Tsue, and P. V. Alberto for valuable discussions. This work was supported by the Foundation for Science and Technology (Portugal) through the Project POCTI/ FIS/451/94.

\section{APPENDIX: RPA TREATMENT OF THE NJL AND EXTENDED $\sigma$ MODELS}

We argue that although the extended NJL model and the extended $\sigma$ model proposed here are equivalent as far as the mean field description of bulk static properties of hadronic matter is concerned, they lead, in the RPA approximation, to manifestly distinct dynamics.

For simplicity, we forget about the $\sigma$ dependence of the coupling coefficient $g_{\sigma}$. The Lagrangian (11) is chiral invariant. Therefore, one of the RPA modes comes at zero energy. This is the pion. Avoiding straightforward algebraic developments, we present the quadratic Hamiltonian which describes the RPA modes having zero momentum,

$$
\begin{aligned}
\mathcal{H}^{(2)}= & \sum_{p_{F}<|k|<\Lambda} \varepsilon_{k}\left(c_{k, \tau}^{\dagger} c_{k, \tau}+d_{k, \tau}^{\dagger} d_{k, \tau}\right) \\
& +m_{\sigma}\left(a_{0}^{\dagger} a_{0}+b_{0}^{0 \dagger} b_{0}^{0}+b_{0}^{+\dagger} b_{0}^{+}+b_{0}^{-\dagger} b_{0}^{-}\right)+\frac{g}{\sqrt{2 m_{\sigma} V}} \\
& \times \sum_{p_{F}<|k|<\Lambda} \frac{|k|}{\varepsilon_{k}}\left(c_{k, \tau}^{\dagger} d_{-k,-\tau}^{\dagger}-c_{k, \tau} d_{-k,-\tau}\right)\left(a_{0}+a_{0}^{\dagger}\right)
\end{aligned}
$$




$$
\begin{aligned}
& +\frac{g}{\sqrt{2 m_{\sigma} V}} \sum_{p_{F}<|k|<\Lambda}\left\{c _ { k , \tau } ^ { \dagger } d _ { - k , - \tau ^ { \prime } } ^ { \dagger } \left[T_{\tau, \tau^{\prime}}^{+}\left(b_{0}^{-\dagger}+b_{0}^{+}\right)\right.\right. \\
& \left.+T_{\tau, \tau^{\prime}}^{-}\left(b_{0}^{+\dagger}+b_{0}^{-}\right)+2 T_{\tau, \tau^{\prime}}^{0}\left(b_{0}^{0 \dagger}+b_{0}^{0}\right)\right]-c_{k, \tau} d_{-k,-\tau^{\prime}} \\
& \times\left[T_{\tau, \tau^{\prime}}^{+}\left(b_{0}^{+\dagger}+b_{0}^{-}\right)+T_{\tau, \tau^{\prime}}^{-}\left(b_{0}^{-\dagger}+b_{0}^{+}\right)\right. \\
& \left.\left.+2 T_{\tau, \tau^{\prime}}^{0}\left(b_{0}^{0 \dagger}+b_{0}^{0}\right)\right]\right\} .
\end{aligned}
$$

Here, $\varepsilon_{k}=\sqrt{k^{2}+M^{2}}, c^{\dagger}, c, d^{\dagger}$, and $d$ are fermion and antifermion operators; $a^{\dagger}$ and $a$ are scalar meson operators; and $b^{0 \dagger}, b^{0}, b^{+\dagger}, b^{+}, b^{-\dagger}$, and $b^{-}$are pseudoscalar meson operators. The quantities $\left(T_{\tau, \tau^{\prime}}^{+}+T_{\tau, \tau^{\prime}}^{-}\right), i\left(T_{\tau, \tau^{\prime}}^{+}-T_{\tau, \tau^{\prime}}^{-}\right)$, and $2 T_{\tau, \tau^{\prime}}^{0}$ are Pauli matrices, or more precisely, they are tensor products of Pauli matrices in isospin times identity matrices in spin, the indices $\tau$ being pairs of spin-isospin indices. Summation over repeated $\tau, \tau^{\prime}$ indices is understood. Terms responsible for the excitation of finite momentum RPA modes have been deliberately omitted. The quantities

$$
\begin{aligned}
& c_{k, \tau}^{\dagger} d_{-k,-\tau}^{\dagger}, \quad c_{k, \tau}^{\dagger} d_{-k,-\tau^{\prime}}^{\dagger} T_{\tau, \tau^{\prime}}^{j}, \\
& d_{-k,-\tau^{\prime}} c_{k, \tau} T_{\tau, \tau^{\prime}}^{j}, \quad j \in\{+,-, 0\}
\end{aligned}
$$

behave as quasibosons. For instance,

$$
\begin{gathered}
{\left[d_{-k,-\tau^{\prime \prime \prime}} c_{k, \tau^{\prime \prime}}, c_{k, \tau}^{\dagger} d_{-k,-\tau^{\prime}}^{\dagger}\right] T_{\tau^{\prime \prime}, \tau^{\prime \prime \prime}}^{j} T_{\tau, \tau^{\prime}}^{k} \approx \delta_{j k} \eta_{k},} \\
\eta_{+}=\eta_{-}=4, \quad \eta_{0}=2 \\
{\left[d_{-k,-\tau^{\prime}} c_{k, \tau^{\prime}}, c_{k, \tau}^{\dagger} d_{-k,-\tau}^{\dagger}\right] \approx 4 .}
\end{gathered}
$$

The result requires replacing such quantities as $d_{-k,-\tau^{\prime}}^{\dagger} d_{-k,-\tau^{\prime \prime \prime}}$ and $c_{k, \tau}^{\dagger} c_{k, \tau^{\prime \prime}}$ by 0 , which is their ground state expectation value. Notice also that

$$
\begin{aligned}
& {\left[c_{k, \tau^{\prime \prime}}^{\dagger} c_{k, \tau^{\prime \prime}}, c_{k, \tau}^{\dagger} d_{-k,-\tau^{\prime}}^{\dagger}\right] T_{\tau, \tau^{\prime}}^{k}} \\
& \quad=\left[d_{-\vec{k},-\tau^{\prime \prime}}^{\dagger} d_{-\vec{k},-\tau^{\prime \prime}}, c_{k, \tau}^{\dagger} d_{-k,-\tau^{\prime}}^{\dagger}\right] T_{\tau, \tau^{\prime}}^{k} \\
& \quad=c_{k, \tau}^{\dagger} d_{-k,-\tau^{\prime}}^{\dagger} T_{\tau, \tau^{\prime}}^{k} .
\end{aligned}
$$

As an example, we present now more explicitly the computation of charged pseudoscalar modes. The following commutators are easily obtained:

$$
\begin{aligned}
{\left[\mathcal{H}^{(2)}, c_{k, \tau}^{\dagger} d_{-k,-\tau^{\prime}}^{\dagger} T_{\tau, \tau^{\prime}}^{+}\right] \approx } & 2 \varepsilon_{k} c_{k, \tau}^{\dagger} d_{-k,-\tau^{\prime}}^{\dagger} T_{\tau, \tau^{\prime}}^{+} \\
& +\frac{4 g}{\sqrt{2 m_{\sigma} V}}\left(b_{0}^{-}+b_{0}^{+\dagger}\right), \\
{\left[\mathcal{H}^{(2)}, c_{k, \tau} d_{-\vec{k},-\tau^{\prime}} T_{\tau, \tau^{\prime}}^{-}\right] \approx } & -2 \varepsilon_{k} c_{\vec{k}, \tau} d_{-k,-\tau^{\prime}} T_{\tau, \tau^{\prime}}^{-} \\
& +\frac{4 g}{\sqrt{2 m_{\sigma} V}}\left(b_{0}^{-}+b_{0}^{+\dagger}\right), \\
{\left[\mathcal{H}^{(2)}, b_{0}^{+\dagger}\right]=m_{\sigma} b_{0}^{+\dagger}+} & \frac{g}{\sqrt{2 m_{\sigma} V}} \sum_{p_{F}<|\vec{k}|<\Lambda} \\
\times & \left(c_{k, \tau}^{\dagger} d_{-k,-\tau^{\prime}}^{\dagger} T_{\tau, \tau^{\prime}}^{+}-c_{k, \tau} d_{-k^{\prime},-\tau} T_{\tau, \tau^{\prime}}^{-}\right), \\
{\left[\mathcal{H}^{(2)}, b_{0}^{-}\right]=-m_{\sigma} b_{0}^{-}-} & \frac{g}{\sqrt{2 m_{\sigma} V}} \sum_{p_{F}<|k|<\Lambda} \\
\times & \left(c_{k, \tau}^{\dagger} d_{-k,-\tau^{\prime}}^{\dagger} T_{\tau, \tau^{\prime}}^{+}-c_{k, \tau} d_{-k,-\tau^{\prime}} T_{\tau, \tau^{\prime}}^{-}\right) .
\end{aligned}
$$

It follows that

$$
\begin{aligned}
& {\left[\mathcal{H}^{(2)}, \sum_{p_{F}<|k|<\Lambda} x_{k}\left(c_{k, \tau}^{\dagger} d_{-k,-\tau^{\prime}}^{\dagger} T_{\tau, \tau^{\prime}}^{+}+c_{k, \tau} d_{-k,-\tau^{\prime}} T_{\tau, \tau^{\prime}}^{-}\right)\right.} \\
& \left.\quad-\left(b_{0}^{-}-b_{0}^{+\dagger}\right)\right] \approx \sum_{p_{F}<|k|<\Lambda}\left(2 x_{k} \varepsilon_{k}+\frac{2 g}{\sqrt{2 m_{\sigma} V}}\right) \\
& \quad \times\left(c_{k, \tau}^{\dagger} d_{-k,-\tau^{\prime}}^{\dagger} T_{\tau, \tau^{\prime}}^{+}-c_{k, \tau} d_{-k,-\tau^{\prime}} T_{\tau, \tau^{\prime}}^{-}\right) \\
& \quad+\left(\sum_{p_{F}<|k|<\Lambda} \frac{8 g x_{k}}{\sqrt{2 m_{\sigma} V}}+m_{\sigma}\right)\left(b_{0}^{-}+b_{0}^{+\dagger}\right)=0 .
\end{aligned}
$$

The result clearly follows from the gap equation, for

$$
x_{k}=-\frac{g}{\sqrt{2 m_{\sigma} V} \varepsilon_{k}} .
$$

This is the RPA pseudoscalar mode, and its eigenfrequency is 0 .

Similarly, we find that the eigenfrequency of the RPA scalar mode is close to $m_{\sigma}$. We present the computation of scalar modes. The following commutators are easily obtained:

$$
\begin{aligned}
{\left[\mathcal{H}^{(2)}, c_{k, \tau}^{\dagger} d_{-k,-\tau}^{\dagger}\right] \approx 2 } & \varepsilon_{k} c_{k, \tau}^{\dagger} d_{-k,-\tau}^{\dagger} \\
& +\frac{4 g}{\sqrt{2 m_{\sigma} V}} \frac{|k|}{\varepsilon_{k}}\left(a_{0}^{-}+a_{0}^{+\dagger}\right), \\
{\left[\mathcal{H}^{(2)}, c_{k, \tau} d_{-k,-\tau}\right] \approx-} & 2 \varepsilon_{k} c_{k, \tau} d_{-k,-\tau} \\
& +\frac{4 g}{\sqrt{2 m_{\sigma} V}} \frac{|k|}{\varepsilon_{k}}\left(a_{0}^{-}+a_{0}^{+\dagger}\right), \\
{\left[\mathcal{H}^{(2)}, a_{0}^{+\dagger}\right]=} & m_{\sigma} a_{0}^{+\dagger}+\frac{g}{\sqrt{2 m_{\sigma} V}} \sum_{p_{F}<|k|<\Lambda} \frac{|k|}{\varepsilon_{k}} \\
& \times\left(c_{k, \tau}^{\dagger} d_{-k,-\tau}^{\dagger}-c_{k, \tau} d_{-k,-\tau}\right), \\
{\left[\mathcal{H}^{(2)}, a_{0}^{-}\right]=} & -m_{\sigma} a_{0}^{-}-\frac{g}{\sqrt{2 m_{\sigma} V}} \sum_{p_{F}<|k|<\Lambda} \frac{|k|}{\varepsilon_{k}} \\
& \times\left(c_{k, \tau}^{\dagger} d_{-k,-\tau}^{\dagger}-c_{k, \tau} d_{-k,-\tau}\right) .
\end{aligned}
$$

It follows that

$$
\begin{aligned}
& {\left[\mathcal{H}^{(2)}, \sum_{p_{F}<|k|<\Lambda}\left(X_{k} c_{k, \tau}^{\dagger} d_{-k,-\tau}^{\dagger}+Y_{k} c_{k, \tau} d_{-k,-\tau}\right)\right.} \\
& \left.-\eta a_{0}^{-}+\xi a_{0}^{+\dagger}\right] \approx \sum_{p_{F}<|k|<\Lambda} 2 \varepsilon_{k}\left(X_{\vec{k}} c_{k, \tau}^{\dagger} d_{-\vec{k},-\tau}^{\dagger}\right. \\
& \left.-Y_{\vec{k}} c_{k, \tau} d_{-k,-\tau}\right)+\sum_{p_{F}<|k|<\Lambda} \frac{(\eta+\xi) g}{\sqrt{2 m_{\sigma} V}} \frac{|k|}{\varepsilon_{k}}\left(c_{k, \tau}^{\dagger} d_{-k,-\tau}^{\dagger}\right. \\
& \left.-c_{k, \tau} d_{-k,-\tau}\right)+\sum_{p_{F}<|k|<\Lambda} \frac{4 g\left(X_{k}+Y_{k}\right)}{\sqrt{2 m_{\sigma} V}} \frac{|k|}{\varepsilon_{k}}\left(a_{0}^{-}+a_{0}^{+\dagger}\right) \\
& +m_{\sigma}\left(\eta a_{0}^{-}+\xi a_{0}^{+\dagger}\right)=\Omega_{s}\left[\sum _ { p _ { F } < | k | < \Lambda } \left(X_{k} c_{k, \tau}^{\dagger} d_{-k,-\tau}^{\dagger}\right.\right. \\
& \left.\left.+Y_{k} c_{k, \tau} d_{-k,-\tau}\right)-\eta a_{0}^{-}+\xi a_{0}^{+\dagger}\right]
\end{aligned}
$$


The RPA equations read

$$
\begin{aligned}
2 \varepsilon_{k} X_{k}+\frac{(\eta+\xi) g}{\sqrt{2 m_{\sigma} V}} \frac{|k|}{\varepsilon_{k}} & =\Omega_{s} X_{k}, \\
2 \varepsilon_{k} Y_{k}+\frac{(\eta+\xi) g}{\sqrt{2 m_{\sigma} V}} \frac{|k|}{\varepsilon_{k}} & =-\Omega_{s} Y_{k}, \\
\sum_{p_{F}<|k|<\Lambda} \frac{4 g\left(X_{k}+Y_{k}\right)}{\sqrt{2 m_{\sigma} V}} \frac{|k|}{\varepsilon_{k}}+m_{\sigma} \xi & =\Omega_{s} \xi, \\
\sum_{p_{F}<|k|<\Lambda} \frac{4 g\left(X_{k}+Y_{k}\right)}{\sqrt{2 m_{\sigma} V}} \frac{|k|}{\varepsilon_{k}}+m_{\sigma} \eta & =-\Omega_{s} \eta,
\end{aligned}
$$

where $X_{k}, Y_{k}, \xi$, and $\eta$ are the RPA amplitudes, and $\Omega_{s}$ is the renormalized $\sigma$ mass

Let $\mathcal{G}_{k}=\frac{2 g}{\sqrt{2 m_{\sigma} V}} \frac{|k|}{\varepsilon_{k}}$. The dispersion relation is obtained as

$$
1=\frac{2 m_{\sigma}}{\Omega^{2}-m_{\sigma}^{2}} \sum_{p_{F}<|k|<\Lambda} \mathcal{G}_{k}^{2} \frac{4 \varepsilon_{k}}{\Omega^{2}-4 \varepsilon_{k}^{2}},
$$

showing that $\Omega_{s} \approx m_{\sigma}<2 M$. Indeed, the left-hand side (1.h.s.) of the dispersion equation becomes infinite for $\Omega^{2}=$ $m_{\sigma}^{2}$ and for $\Omega^{2}=4 \varepsilon_{k}^{2}$, changing sign at each place. The zero $\Omega_{s}^{2}$ lies slightly below $m_{\sigma}^{2}$. Between $m_{\sigma}^{2}$ and $4 M^{2}$, the 1.h.s. of the dispersion equation is negative. Between $4 M^{2}+4 p_{F}^{2}$ and $4 M^{2}+\Lambda^{2}$, we find a continuum of Landau damped modes.

Next, we consider the quadratic Hamiltonian which, for the NJL model, describes the RPA modes having zero momentum,

$$
\begin{aligned}
\mathcal{H}_{\mathrm{NJL}}^{(2)}= & \sum_{p_{F}<k<\Lambda} \varepsilon_{k}\left(c_{k, \tau}^{\dagger} c_{k, \tau}+d_{k, \tau}^{\dagger} d_{k, \tau}\right)-2 G_{s} \sum_{p_{F}<|k|,\left|k^{\prime}\right|<\Lambda} \\
& \times\left[\frac { | k | } { \varepsilon _ { k } } ( c _ { k , \tau } ^ { \dagger } d _ { - k , - \tau } ^ { \dagger } - c _ { k , \tau } d _ { - k , - \tau } ) \frac { | k ^ { \prime } | } { \varepsilon _ { k } ^ { \prime } } \left(c_{k^{\prime}, \tau^{\prime}}^{\dagger} d_{-k^{\prime},-\tau^{\prime}}^{\dagger}\right.\right. \\
& \left.-c_{k^{\prime}, \tau^{\prime}} d_{-k^{\prime},-\tau^{\prime}}\right)+\left(c_{k, \tau}^{\dagger} d_{-k^{\prime},-\tau^{\prime}}^{\dagger} T_{\tau, \tau^{\prime}}^{+}\right. \\
& \left.-c_{k, \tau} d_{-k,-\tau^{\prime}} T_{\tau, \tau^{\prime}}^{-}\right)\left(c_{k^{\prime}, \tau^{\prime \prime}}^{\dagger} d_{-k^{\prime},-\tau^{\prime \prime \prime}}^{\dagger} T_{\tau^{\prime \prime}, \tau^{\prime \prime \prime}}^{-}\right. \\
& \left.-c_{k^{\prime}, \tau^{\prime \prime}} d_{-k^{\prime},-\tau^{\prime \prime \prime}} T_{\tau^{\prime \prime}, \tau^{\prime \prime \prime}}^{+}\right)+\left(c_{k, \tau}^{\dagger} d_{-k,-\tau^{\prime}}^{\dagger} T_{\tau, \tau^{\prime}}^{-}\right. \\
& \left.-c_{k, \tau} d_{-k,-\tau^{\prime}} T_{\tau, \tau^{\prime}}^{+}\right)\left(c_{k^{\prime}, \tau^{\prime \prime}}^{\dagger} d_{-k^{\prime},-\tau^{\prime \prime \prime}}^{\dagger} T_{\tau^{\prime \prime}, \tau^{\prime \prime \prime}}^{+}\right. \\
& \left.-c_{k^{\prime}, \tau^{\prime \prime}} d_{-k^{\prime},-\tau^{\prime \prime \prime}} T_{\tau^{\prime \prime}, \tau^{\prime \prime \prime}}^{-}\right)+2\left(c_{k, \tau}^{\dagger} d_{-k,-\tau^{\prime}}^{\dagger} T_{\tau, \tau^{\prime}}^{0}\right. \\
& \left.-c_{k, \tau} d_{-k,-\tau^{\prime}} T_{\tau, \tau^{\prime}}^{0}\right)\left(c_{k^{\prime}, \tau^{\prime \prime}}^{\dagger} d_{-k^{\prime},-\tau^{\prime \prime \prime}}^{\dagger} T_{\tau^{\prime \prime}, \tau^{\prime \prime \prime}}^{0}\right. \\
& \left.\left.-c_{k^{\prime}, \tau^{\prime \prime}} d_{-k^{\prime},-\tau^{\prime \prime \prime}} T_{\tau^{\prime \prime}, \tau^{\prime \prime \prime}}^{0}\right)\right]
\end{aligned}
$$

The following commutators are easily obtained:

$$
\begin{aligned}
& {\left[\mathcal{H}_{\mathrm{NJL}}^{(2)}, c_{k, \tau}^{\dagger} d_{-k,-\tau^{\prime}}^{\dagger} T_{\tau, \tau^{\prime}}^{+}\right]} \\
& \approx 2 \varepsilon_{k} c_{k, \tau}^{\dagger} d_{-k,-\tau^{\prime}}^{\dagger} T_{\tau, \tau^{\prime}}^{+}-16 G_{s} \sum_{p_{F}<\left|k^{\prime}\right|<\Lambda}\left(c_{k^{\prime}, \tau^{\prime \prime}}^{\dagger} d_{-k^{\prime},-\tau^{\prime \prime \prime}}^{\dagger} T_{\tau^{\prime \prime}, \tau^{\prime \prime \prime}}^{+}\right. \\
& \left.\quad-c_{k^{\prime}, \tau^{\prime \prime}} d_{-k^{\prime},-\tau^{\prime \prime \prime}} T_{\tau^{\prime \prime}, \tau^{\prime \prime \prime}}^{-}\right), \\
& \quad\left[\mathcal{H}_{\mathrm{NJL}}^{(2)}, c_{k, \tau} d_{-k,-\tau^{\prime}} T_{\tau, \tau^{\prime}}^{-}\right] \\
& \quad-2 \varepsilon_{k} c_{k, \tau} d_{-k,-\tau^{\prime}} T_{\tau, \tau^{\prime}}^{-}-16 G_{s} \sum_{p_{F}<\left|k^{\prime}\right|<\Lambda}\left(c_{k^{\prime}, \tau^{\prime \prime}}^{\dagger} d_{-k^{\prime},-\tau^{\prime \prime \prime}}^{\dagger} T_{\tau^{\prime \prime}, \tau^{\prime \prime \prime}}^{+}\right. \\
& \left.\quad-c_{k^{\prime}, \tau^{\prime \prime}} d_{-k^{\prime \prime \prime},-\tau^{\prime}} T_{\tau^{\prime \prime}, \tau^{\prime \prime}}^{-}\right) .
\end{aligned}
$$

Thus,

$$
\begin{aligned}
& {\left[\mathcal{H}_{\mathrm{NJL}}^{(2)}, \sum_{p_{F}<|k|<\Lambda} \frac{1}{2 \varepsilon_{k}}\left(c_{k, \tau}^{\dagger} d_{-k,-\tau^{\prime}}^{\dagger} T_{\tau, \tau^{\prime}}^{+}+c_{k, \tau} d_{-k,-\tau^{\prime}} T_{\tau, \tau^{\prime}}^{-}\right)\right]} \\
& \approx\left(1-\sum_{p_{F}<\left|k^{\prime}\right|<\Lambda} \frac{16 G_{s}}{\varepsilon_{k^{\prime}}}\right) \sum_{p_{F}<|k|<\Lambda} \\
& \times\left(c_{k, \tau}^{\dagger} d_{-k^{\prime},-\tau^{\prime}}^{\dagger} T_{\tau, \tau^{\prime}}^{+}-c_{k, \tau} d_{-k,-\tau^{\prime}} T_{\tau, \tau^{\prime}}^{-}\right)=0 .
\end{aligned}
$$

This is the pion mode. Notice that the NJL gap equation reads

$$
1-\sum_{p_{F}<|k|<\Lambda} \frac{16 G_{s}}{\varepsilon_{k}}=0,
$$

and $G_{s}>0$.

We present next the computation of scalar modes. The following commutators are easily obtained:

$$
\begin{aligned}
{\left[\mathcal{H}_{\mathrm{NJL}}^{(2)}, c_{k, \tau}^{\dagger} d_{-k,-\tau}^{\dagger}\right] \approx } & 2 \varepsilon_{k} c_{k, \tau}^{\dagger} d_{-k,-\tau}^{\dagger}-16 G_{s} \frac{|k|}{\varepsilon_{k}} \sum_{p_{F}<\left|k^{\prime}\right|<\Lambda} \\
& \times \frac{\left|k^{\prime}\right|}{\varepsilon_{k^{\prime}}}\left(c_{k^{\prime}, \tau^{\prime}}^{\dagger} d_{-k^{\prime},-\tau^{\prime}}^{\dagger}-c_{k^{\prime}, \tau^{\prime}} d_{-k^{\prime},-\tau^{\prime}}\right), \\
{\left[\mathcal{H}_{\mathrm{NJL}}^{(2)}, c_{k, \tau} d_{-k,-\tau}\right] \approx } & -2 \varepsilon_{k} c_{k, \tau} d_{-k,-\tau}-16 G_{s} \frac{|k|}{\varepsilon_{k}} \sum_{p_{F}<\left|k^{\prime}\right|<\Lambda} \\
& \times \frac{\left|k^{\prime}\right|}{\varepsilon_{k^{\prime}}}\left(c_{\vec{k}^{\prime}, \tau^{\prime}}^{\dagger} d_{-\vec{k}^{\prime},-\tau^{\prime}}^{\dagger}-c_{k^{\prime}, \tau^{\prime}} d_{-k^{\prime},-\tau^{\prime}}\right) .
\end{aligned}
$$

It follows that

$$
\begin{aligned}
& {\left[\mathcal{H}_{\mathrm{NJL}}^{(2)}, \sum_{p_{F}<|k|<\Lambda}\left(X_{k} c_{k, \tau}^{\dagger} d_{-k,-\tau}^{\dagger}+Y_{k} c_{k, \tau} d_{-k,-\tau}\right)\right]} \\
& \approx \sum_{p_{F}<|k|<\Lambda} 2 \varepsilon_{k}\left(X_{k} c_{k, \tau}^{\dagger} d_{-k,-\tau}^{\dagger}-Y_{k} c_{k, \tau} d_{-k,-\tau}\right) \\
& -16 G_{s} \sum_{p_{F}<|k|<\Lambda}\left(X_{k}+Y_{k}\right) \frac{|k|}{\varepsilon_{k}} \\
& \times \sum_{p_{F}<\left|k^{\prime}\right|<\Lambda} \frac{\left|k^{\prime}\right|}{\varepsilon_{k^{\prime}}}\left(c_{k^{\prime}, \tau^{\prime}}^{\dagger} d_{-k^{\prime},-\tau^{\prime}}^{\dagger}-c_{k^{\prime}, \tau^{\prime}} d_{-k^{\prime},-\tau^{\prime}}\right) \\
& =\Omega_{s} \sum_{p_{F}<|k|<\Lambda}\left(X_{k} c_{k, \tau}^{\dagger} d_{-k,-\tau}^{\dagger}+Y_{k} c_{k, \tau} d_{-k,-\tau}\right) .
\end{aligned}
$$

The RPA equations read

$$
\begin{aligned}
& 2 \varepsilon_{k} X_{\vec{k}}-16 G_{s} \frac{|k|}{\varepsilon_{k}} \sum_{p_{F}<\left|k^{\prime}\right|<\Lambda}\left(X_{k^{\prime}}+Y_{k^{\prime}}\right) \frac{\left|k^{\prime}\right|}{\varepsilon_{k^{\prime}}}=\Omega_{s} X_{k}, \\
& 2 \varepsilon_{k} Y_{k}-16 G_{s} \frac{|k|}{\varepsilon_{k}} \sum_{p_{F}<\left|k^{\prime}\right|<\Lambda}\left(X_{k^{\prime}}+Y_{k^{\prime}}\right) \frac{\left|k^{\prime}\right|}{\varepsilon_{k^{\prime}}}=-\Omega_{s} Y_{k},
\end{aligned}
$$

where $X_{k}, Y_{k}$ are the RPA amplitudes, and $\Omega_{s}$ is the renormalized $\sigma$ mass. The dispersion relation is obtained as

$$
1=-16 G_{s} \sum_{p_{F}<|k|<\Lambda} \frac{k^{2}}{\varepsilon_{k}^{2}} \frac{4 \varepsilon_{k}}{\Omega^{2}-4 \varepsilon_{k}^{2}},
$$


showing that $\Omega_{s}=2 M$. Indeed, replacing $\Omega^{2}$ by $4 M^{2}$ in the 1.h.s. of the dispersion equation, we obtain an identity, in view of the NJL gap equation,

$$
1=-16 G_{s} \sum_{p_{F}<|k|<\Lambda} \frac{k^{2}}{\varepsilon_{k}^{2}} \frac{4 \varepsilon_{k}}{4 m^{2}-4 \varepsilon_{k}^{2}}
$$

$$
=-16 G_{s} \sum_{p_{F}<|k|<\Lambda} \frac{k^{2}}{\varepsilon_{k}^{2}} \frac{4 \varepsilon_{k}}{-4 k^{2}}=1 .
$$

In conclusion, we have shown that both models generate pseudoscalar modes with zero mass. However, the $\sigma$ model generates a scalar mode with mass close to $m_{\sigma}$, while the NJL model for nucleons generates a scalar mode with an unacceptable mass, equal to $2 M$.
[1] A. Chodos, R. L. Jaffe, K. Johnson, C. B. Thorn, and V. F. Weisskopf, Phys. Rev. D 9, 3471 (1974).

[2] G. 't Hooft, Phys. Rev. D 14, 3432 (1976).

[3] S. P. Klevansky, Rev. Mod. Phys. 64, 649 (1992).

[4] J. da Providência, M. C. Ruivo, and C. A. de Sousa, Phys. Rev. D 36, 1882 (1987).

[5] J. Carlson, J. Kogut, and V. R. Pandharipande, Phys. Rev. D 27, 233 (1983); R. Sommer and J. Wosiek, Nucl. Phys. B267, 531 (1986).

[6] P. A. M. Guichon, Phys. Lett. B200, 235 (1988).

[7] S. Fleck, W. Benz, K. Shimizu, and K. Yazaki, Nucl. Phys. A510, 731 (1990).

[8] M. Uehara and H. Kondo, Prog. Theor. Phys. 71, 1303 (1984).

[9] B. D. Serot and J. D. Walecka, Adv. Nucl. Phys. 16, 1 (1986); Int. J. Mod. Phys. E 16, 515 (1997).

[10] H. Bohr, in Proceedings of the International Workshop "Fission Dynamics of Atomic Clusters and Nuclei," edited by J. da Providência, D. Brink, F. F. Karpeshin, and F. B. Malik (World Scientific, Singapore, 2001), p. 65.
[11] H. R. Petry, H. Hofestädt, S. Merk, K. Bleuler, H. Bohr, and K. S. Narain, Phys. Lett. B159, 363 (1985).

[12] S. A. Moszkowski (private communication).

[13] T. J. Bürvenich and D. G. Madland, Nucl. Phys. A729, 769 (2003).

[14] V. Koch, T. S. Biro, J. Kunz, and U. Mosel, Phys. Lett. B185, 1 (1987).

[15] G. A. Lalazissis, J. König, and P. Ring, Phys. Rev. C 55, 540 (1997).

[16] K. Rajagopal and F. Wilczek, Phys. Rev. Lett. 86, 3492 (2001); M. Alford, K. Rajagopal, S. Reddy, and F. Wilczek, Phys. Rev. D 64, 074017 (2001); M. G. Alford, Annu. Rev. Nucl. Part. Sci. 51, 131 (2001).

[17] M. Buballa, preprint, Phys. Rept. 407, 205 (2005).

[18] G. Chanfray, M. Ericson, and P. A. M. Guichon, Phys. Rev. C 63, 055202 (2001); G. Chanfray and M. Ericson, submitted to Phys. Rev. C, arXiv:nucl-th/0402018.

[19] N. K. Glendenning, Compact Stars (Springer, New York, 2000). 